Hohlraum Target Alignment from X-ray Detector Images using Starburst Design Patterns

R. R. Leach, A. Conder, O. Edwards, J. Kroll, B. Kozioziemski, E. Mapoles, D. McGuigan, K. Wilhelmsen

January 3, 2011

SPIE Photonics West

San Francisco, CA, United States

January 22, 2011 through January 27, 2011 
This document was prepared as an account of work sponsored by an agency of the United States government. Neither the United States government nor Lawrence Livermore National Security, LLC, nor any of their employees makes any warranty, expressed or implied, or assumes any legal liability or responsibility for the accuracy, completeness, or usefulness of any information, apparatus, product, or process disclosed, or represents that its use would not infringe privately owned rights. Reference herein to any specific commercial product, process, or service by trade name, trademark, manufacturer, or otherwise does not necessarily constitute or imply its endorsement, recommendation, or favoring by the United States government or Lawrence Livermore National Security, LLC. The views and opinions of authors expressed herein do not necessarily state or reflect those of the United States government or Lawrence Livermore National Security, LLC, and shall not be used for advertising or product endorsement purposes. 


\title{
Hohlraum Target Alignment from X-ray Detector Images using Starburst Design Patterns
}

\author{
Richard R. Leach Jr., Alan Conder, Oliver Edwards, Jeremy Kroll, Bernard Kozioziemski, \\ Evan Mapoles, Dave McGuigan, and Karl Wilhelmsen \\ Integrated Computer Control System, National Ignition Facility \\ Laser Science Engineering and Operations, \\ Lawrence Livermore National Laboratory, Livermore, CA. 94551 \\ e-mail: leach1@1lnl.gov
}

\begin{abstract}
National Ignition Facility (NIF) is a high-energy laser facility comprised of 192 laser beams focused with enough power and precision on a hydrogen-filled spherical, cryogenic target to initiate a fusion reaction. The target container, or hohlraum, must be accurately aligned to an x-ray imaging system to allow careful monitoring of the frozen fuel layer in the target. To achieve alignment, $\mathrm{x}$-ray images are acquired through starburst-shaped windows cut into opposite sides of the hohlraum. When the hohlraum is in alignment, the starburst pattern pairs match nearly exactly and allow a clear view of the ice layer formation on the edge of the target capsule. During the alignment process, x-ray image analysis is applied to determine the direction and magnitude of adjustment required. X-ray detector and source are moved in concert during the alignment process. The automated pointing alignment system described here is both accurate and efficient. In this paper, we describe the control and associated image processing that enables automation of the starburst pointing alignment.
\end{abstract}

Key words: NIF, internal confinement fusion, hohlraum, pointing alignment, starburst pattern, ablation, $\mathrm{x}$-ray source and detector, frozen fuel layer

\section{INTRODUCTION}

The National Ignition Facility at the Lawrence Livermore National Laboratory has been operational since 2009 for the study of high-energy density and fusion science. Our first goal is that of achieving ignition, which is to produce a net energy gain for the first time in a laboratory setting [1-3]. To achieve this goal, we have built a $1.8 \mathrm{MJ}$ ultraviolet laser system consisting of 192 beams, capable of delivering up to 500 trillion watts of power onto a $2 \mathrm{~mm}$ diameter target. The target is a spherical capsule that is filled with deuterium-tritium (DT) gas. The surface of the capsule is cooled to form 70 microns of DT ice on the inner surface of the capsule. The target is housed in a cylindrical gold case called a hohlraum. Starburst-shaped viewing windows are etched out of the hohlraum through which $\mathrm{x}$-rays are passed in order to view the capsule and ice layer inside.

Hohlraums act as radiation enclosures that convert the optical laser light into soft $\mathrm{x}$-rays. The radiation field compresses the fusion capsule by $\mathrm{x}$-ray ablation of the outer shell. The ablation process compresses the cryogenically prepared solid DT fuel layer in a spherical rocket implosion. In the final stages, the fuel reaches densities that are 1000 times that of lead, and the central hot spot temperatures will approach 100 million Kelvin $(\mathrm{K})$ to initiate a thermonuclear burn process as occurs naturally in the interiors of the stars [4-5].

Hohlraum alignment is critical in order to achieve fusion, and to monitor the condition of the target capsule and its DT ice layer. The uniformity of the DT ice layer of fusion targets during the cooling process to $18 \mathrm{~K}$ must be carefully controlled [6]. The roughness and position of the solid/vapor interface of the DT fuel inside of the opaque hohlraum can only be seen by low energy x-ray transmission imaging through the aligned starburst windows. This alignment is carried out just outside the NIF target chamber as described in the next section. 


\section{NIF CRYOGENIC TARGET SYSTEM}

The NIF cryogenic target system has three subsystems: 1) the target positioning system, 2) the target characterization system, and 3) the cryogenic system. An illustration of the system is shown in Figure 1. A more complete system description can be found in [7].

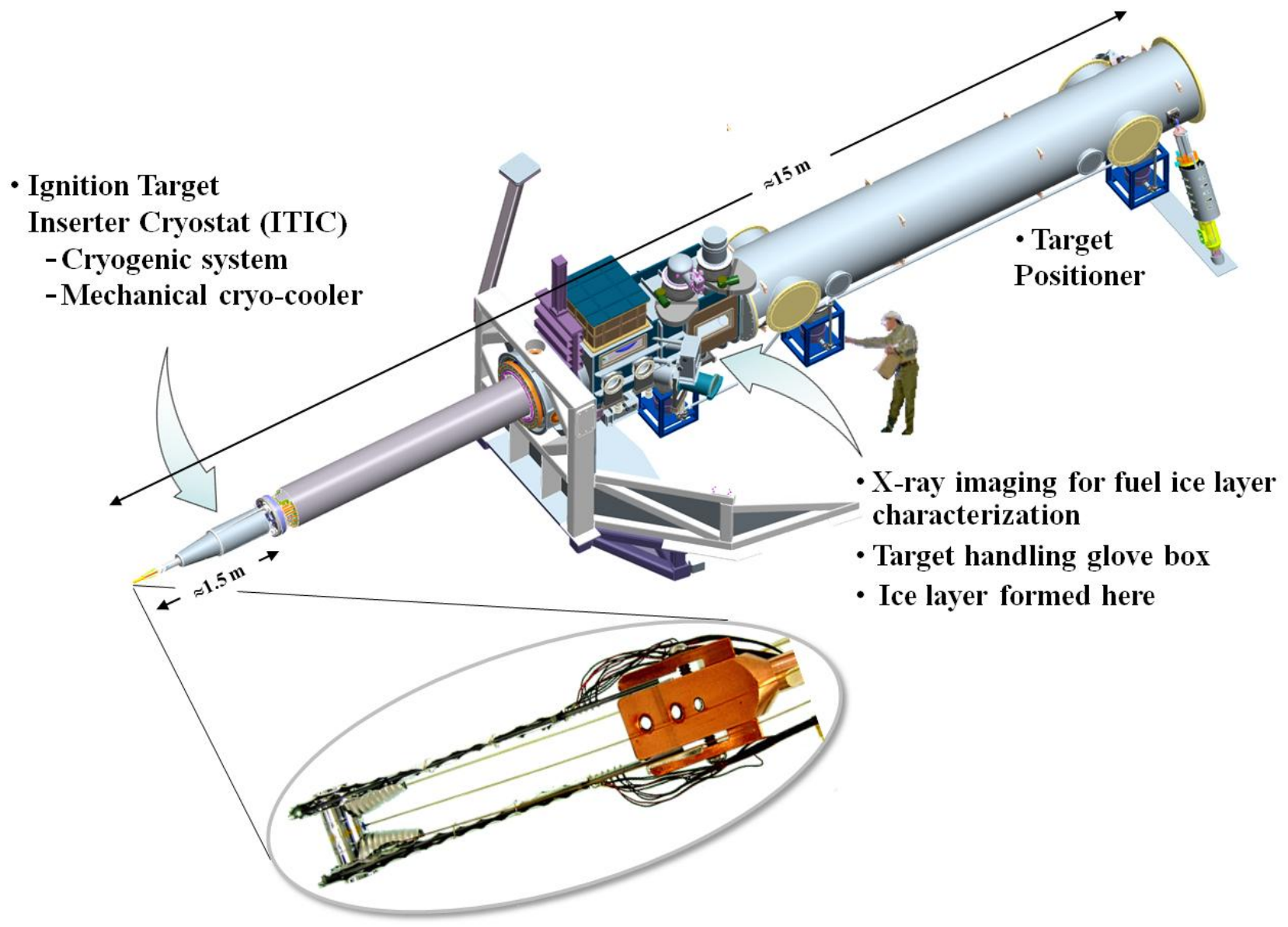

Figure 1. The ignition target hohlraum assembly shown at the tip of the cryogenic target system is designed to be compatible with vacuum, 5e-6 Torr, and a temperature of $18 \mathrm{~K}$.

The NIF ignition target is composed of a cryogenic base, which provides the electrical, thermal and gas fill connections to an ignition target inserter cryostat and an ignition hohlraum assembly. The target base contains a reservoir assembly that carries the DT, filling the capsule prior to production of an ice layer. The ignition hohlraum design contains starburst windows for viewing the ice layer, which forms on the inner surface of the target capsule (Figure 2). 


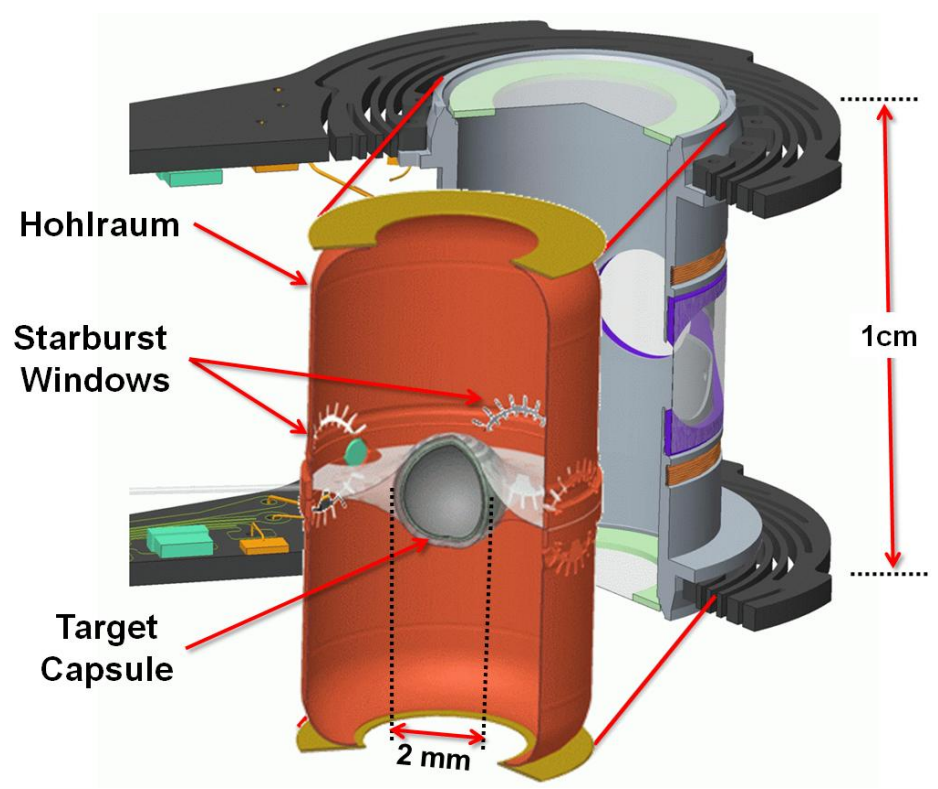

Figure 2. Cryogenic target capsule $(2 \mathrm{~mm})$ inside the hohlraum $(1 \mathrm{~cm}$ height) is held in place using an $110 \mathrm{~nm}$ Formvar tent during alignment to stabilize the view for evaluation of target's frozen surface.

The target capsule is centered inside a hohlraum cylinder measuring just ten millimeters high by five millimeters in diameter (Figure 2). In radiation thermodynamics, a hohlraum is defined as "a cavity whose walls are in radiative equilibrium with the radiant energy within the cavity." The hohlraum is made from a high-atomic-number material (an element with a high electron density) such as gold and uranium. The starburst pattern contains two non-symmetric (upper and lower) ports, as shown in Figure 3. At the bottom is a liquid meniscus viewing window used during capsule filling and at the top is a wider window section to view the final growth of the DT ice. Note the trapezoid shaped meniscus viewing window in Figure 3, which is also used in the alignment image processing algorithm.
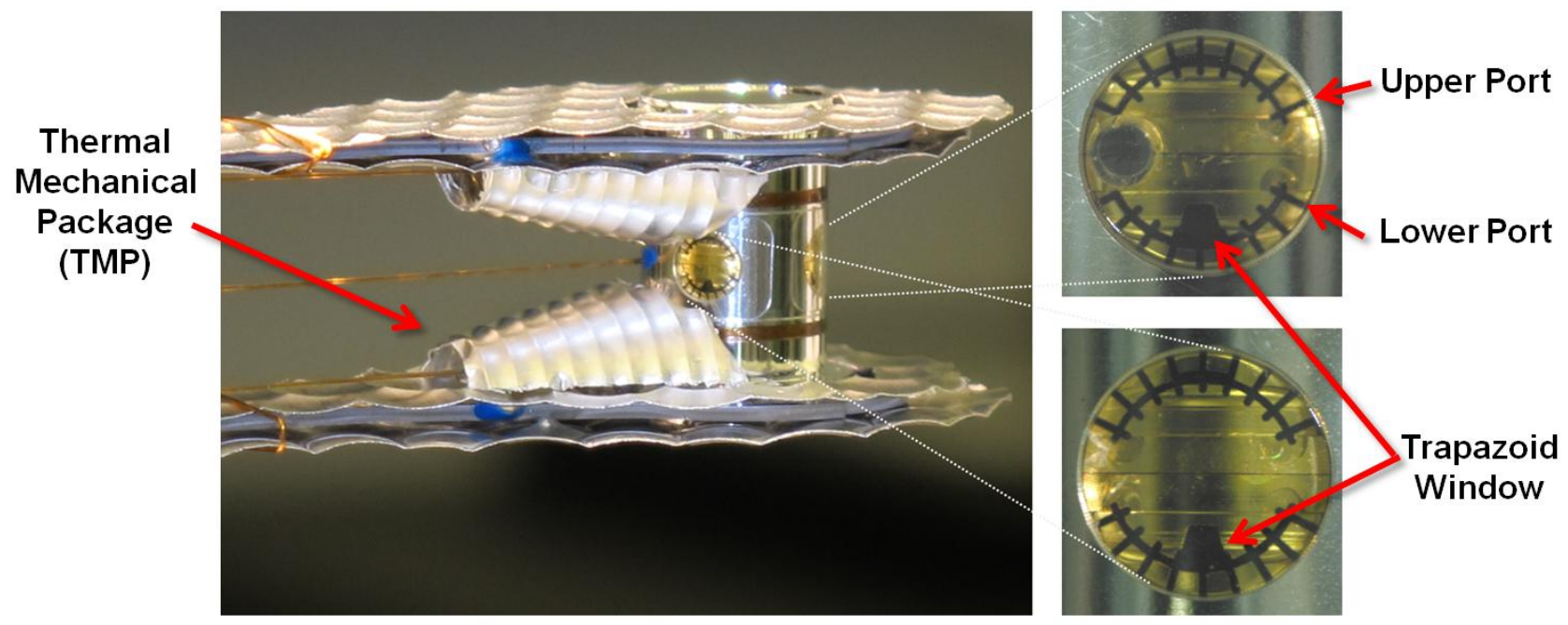

Figure 3 Alignment for $x$-ray imaging of ice layer is accomplished using starburst side views of two, separate, orthogonal starburst window pairs.

The geometric shape of the DT ice is determined by the details of the heat transfer between the hohlraum and the capsule. Precise control of this heat transfer is needed to form a spherical ice layer in the capsule centered in the cylindrical hohlraum. This control is achieved through the integration of a thermal-mechanical package (TMP) with the 
hohlraum and capsule assembly. The TMP consists of two precision-fabricated aluminum shells joined by a precisionfabricated band with cutouts to accommodate diagnostics requirements.

\section{HOHLRAUM ALIGNMENT}

The cryogenic target positioning system (CTS) consists of three main parts. The target positioning boom is based on the existing target positioner attached to the NIF target chamber. The ignition target inserter cryostat is attached to the end of the target positioner and cools the target and the fuel mixture to meet temperature and uniformity requirements. This system has the cooling capacity necessary to maintain the assembly at $18 \mathrm{~K}$, as well as the mechanical stability to accurately position the hohlraum to within ten microns of the center of the NIF target chamber at shot time. The third part is a layering and characterization station where the D-T fuel layer is characterized along three axes. Alignment for the layering and characterization is done using control loop and image processing software. Proper alignment requires a series of control loops to position the hardware for x-ray imaging.

\subsection{Loop control software}

Alignment of the hohlraum is done using position commands to the x-ray source and camera, which are mounted on opposite sides of the hohlraum starburst window. Precise alignment occurs when the front and rear starburst cut-outs are in perfect alignment between the $\mathrm{x}$-ray source and the x-ray camera. Control loop operations for starburst positioning are carried out through a series of iterations that first estimate the current alignment of the hohlraum and then issue a command to adjust the position of the hohlraum. As shown in the diagram in Figure 4, the x-ray source and camera assembly is lowered in place just outside the target chamber where the alignment process is done. A set of 20-30 x-ray images takes approximately 2 minutes to complete. Target stability during each frame is under $2 \mu \mathrm{m}$ p-p.

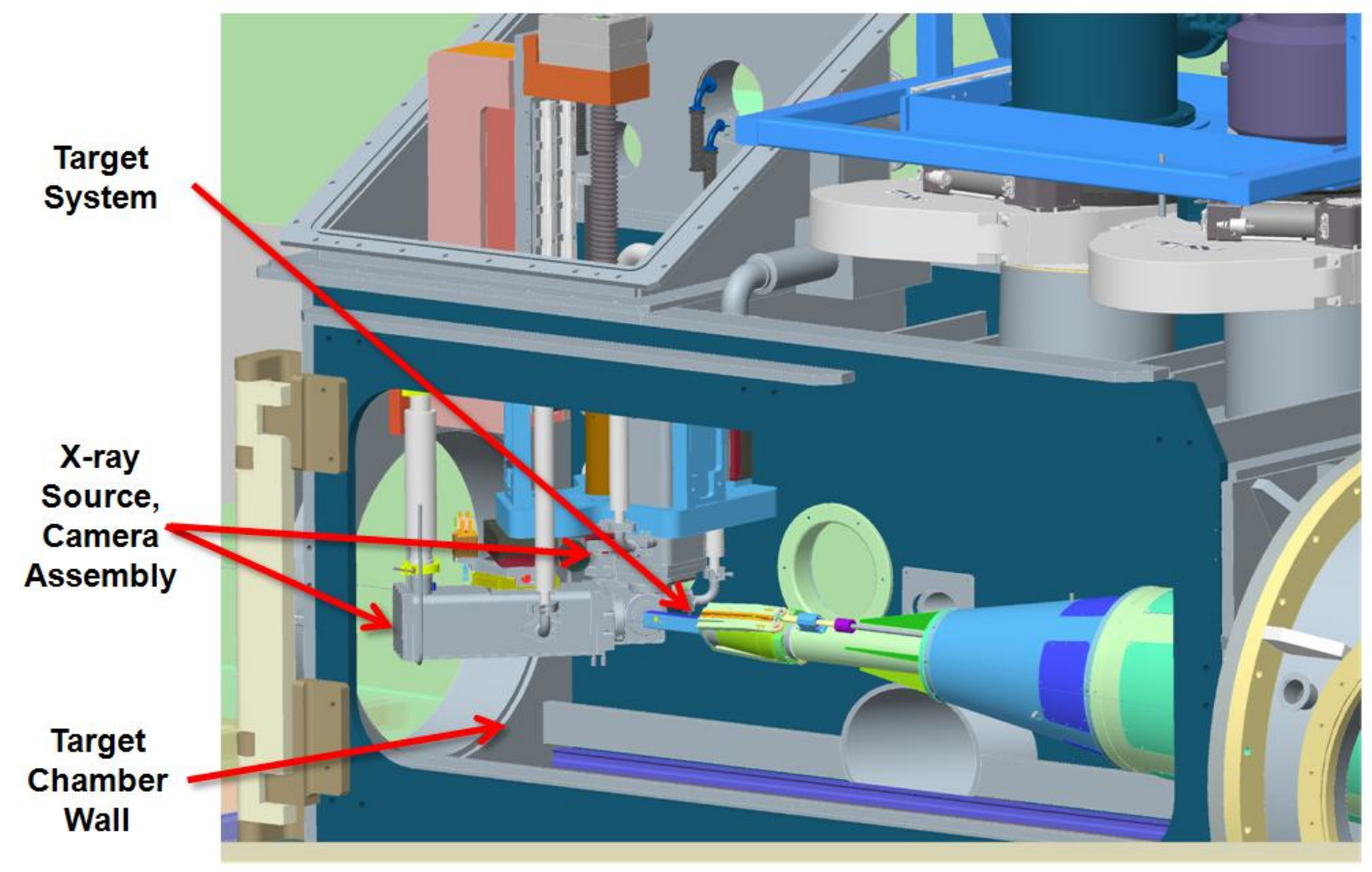

Figure 4. X-ray images are taken just outside the target chamber during the alignment process. A set of 20-30 images, each requiring 4-6 seconds, are acquired.

\subsection{Loop command sequence}

A sequence of software commands is executed during alignment. The control loop software sets up and collects an $\mathrm{x}$ ray image of the hohlraum front and rear starburst overlap. From this image, the image processing software estimates the alignment error magnitude for both vertical and horizontal movements. 
Since the cause of obstructing overlap between the front and rear starburst cut-outs is not deterministic, movement can only be estimated in magnitude. Therefore, to align the x-ray source and camera about the hohlraum, the direction of movement, both horizontal and vertical, must be determined.

To determine these parameters, the control loop moves the hohlraum in four separate loop tests. These loop tests are the combinations of positive and negative moves for both the horizontal and vertical direction using the estimated magnitude. For each of the four moves, the area of the front to rear starburst window superposition is measured. The best test move is selected based on the loop with the largest area. The control loop then repositions the hohlraum to this location. If the remaining error magnitude is within tolerance, the control loop is defined as aligned. If not, another set of four test moves is preformed and tested. This alignment sequence is performed on the three orthogonal views of the hohlraum to provide a complete view of the DT ice layer with minimal obstruction.

\section{IMAGE PROCESSING}

When x-ray images are acquired during hohlraum alignment, a wide range of possible patterns can result from the superimposed front and rear starburst windows. The kaleidoscope of patterns that occur as the hohlraum moves in and out of alignment makes it difficult to identify a consistent pattern or feature to key on (Figure 5). Integration of the light in the image is also problematic due to asymmetries and local minima. Noise, systematic changes, time constraints, computational expense, complexity, and repeatability provide additional challenges.

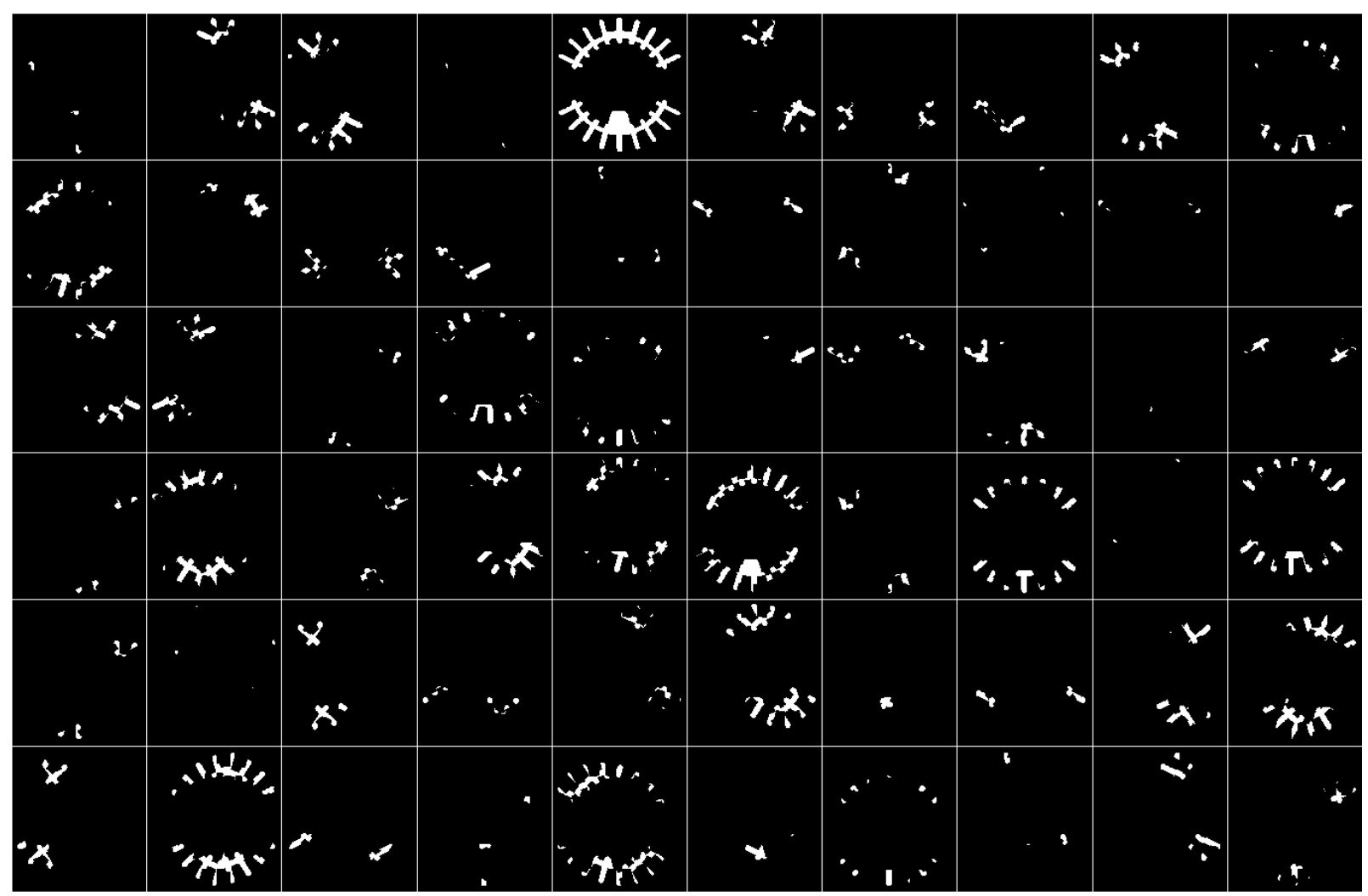

Figure 5. A montage of 60 randomly generated initial positions for the hohlraum alignment are shown. The kaleidoscope of patterns that occur as the hohlraum moves in and out of alignment can vary greatly. The fifth image from the left on the top row shows near-perfect alignment.

To illustrate the variety of patterns that can occur when the hohlraum is misaligned, Figure 5 displays a montage of 60 randomly generated simulated hohlraum image patterns where each tile in the montage represents a unique initial condition of the hohlraum alignment. X-rays only penetrate when both front and rear starburst windows are aligned. If the hohlraum is not initially close to optimal alignment, very little of the starburst patterns is visible in the image. 
To provide automated alignment of superimposed starburst patterns from x-ray hohlraum images, we attempt to find and use distinct features to generally provide reference points for any given starburst alignment configuration. Often there is no general or exact definition of what constitutes a feature for a given image processing task. In many cases it has to be discovered by careful examination of the images.

\subsection{Starburst pattern center estimation}

One of the most common and studied features in image processing involves finding edges first suggested by Marr [8]. The starburst pattern contains somewhat distinct edges. Locating the edges of the hohlraum opening accurately would provide a good estimate of the hohlraum center $\left(\mathrm{x}_{\mathrm{C}^{2}} \mathrm{y}_{\mathrm{C}}\right)$.

The standard approach is to define an edge as step discontinuities in the image signal. Then the edges can be located simply by finding the local maxima of the derivative of the image or using the zero-crossing points of the second derivative. This approach was later developed by Marr and Hildreth [9], Canny [10,11] and others [12, 13] where the gradient of the image $I$ is given by the vector

The magnitude of this gradient is given by

$$
\left.\nabla I=\left[\frac{\partial I}{\partial x^{x}} \frac{\partial I}{\partial y}\right]\right]^{x}
$$

And the direction of the gradient is given by

$$
\sqrt{\left(\frac{\partial n}{\partial x}\right)^{2}+\left(\frac{\partial n}{\partial y}\right)^{2}}
$$

$$
\tan ^{-1}\left(\frac{\partial I}{\partial y} / \frac{\partial n}{\partial x}\right)
$$

The gradient vector can then be calculated using one of several common operators such as Robert's Cross, Prewitt, and Sobel, and other techniques [14].

Several problems arise from this approach, however. Noise is often a major problem dealing with differential edge detection. Spikes resulting from taking the derivative of the image can easily mask the location of the true edges. If there is variation in the noise levels from image to image, repeatability suffers and both false positives and false negatives increase. If we smooth the image, however the effects of noise can be reduced.

Smoothing is done by first forming mean intensity profile vectors for all rows and all columns in the image given by

and

$$
\overline{\mathrm{X}}=\frac{1}{\mathrm{~N}} \sum_{1}^{\mathrm{N}} \mathrm{X}_{\mathrm{i}}
$$

$$
\overline{\mathrm{y}}=\frac{1}{\mathrm{M}} \Sigma_{1}^{\mathrm{M}} \mathrm{Y}_{\mathrm{i}}
$$

The resulting vectors are then used to calculate the initial center estimate of the starburst pattern as shown in Figure 6. There is an additional advantage to this approach due to the reduction of computation time using 1D vector calculations. The center estimate measurement $\left(\mathrm{x}_{\mathrm{C}}, \mathrm{y}_{\mathrm{C}}\right)$ is used as the first reference point in the image.

\subsection{Binary threshold from noise measurements}

The strongest intensities lie in the areas of the image where the front and rear starburst patterns are aligned and allow xrays to pass to the camera. At this point, a simple and direct approach is to binarize the image. Finding a reliable threshold for this step is difficult, however, with greatly varying levels of signal and noise in images. In addition, as seen in Figure 6, non-uniformity of x-ray energy within the window due to target absorption and other effects increase error in locating the starburst window in its entirety. Thresholding can be extremely sensitive when developing an automatic procedure for determining thresholds that apply to all images. 
We can, however, again take advantage of the starburst pattern symmetry. The center estimate is particularly helpful and allows us to define a consistent noise region in the image using $y_{\mathbb{C}}$ and the nominal size of the starburst window pattern. Figure 7 (in the upper-right box) illustrates horizontal noise bands or strips that are used to determine the minimum, maximum, standard error, and mean of the noise. Twice the noise standard error added to the noise maximum provides a reliable threshold for image binarization. During this operation, small objects are filtered out such as spurs and lone pixels. Afterwards, the center estimate for $\left(\mathrm{x}_{\mathrm{c}}, \mathrm{y}_{\mathrm{C}}\right)$ is updated as described in the previous section to provide a more accurate center estimate.

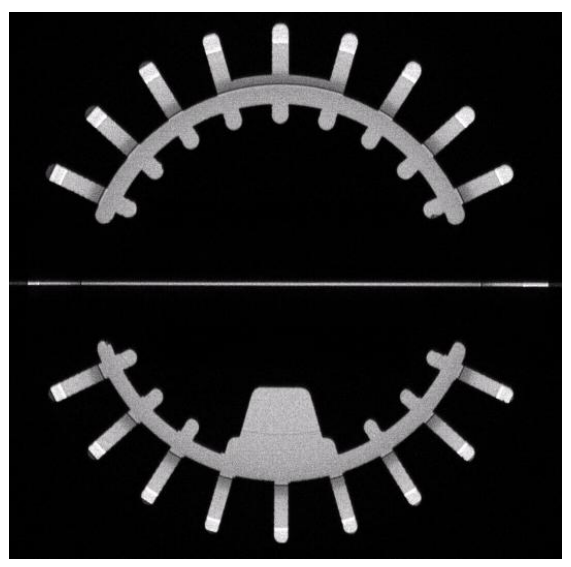

Figure 6. Starburst $x$-ray image. Diffraction by the edge of the target capsule is visible in the upper and lower parts. Horizontal strips above and below the horizontal line in the center are used for noise background measurements. The trapezoidal meniscus opening (bottom, center) is used for alignment.

\subsection{Selecting pattern features for offset measurements}

The final stage of the process involves selecting features in the image and measuring their offset from the center $\left(\mathrm{x}_{\mathrm{E} s} \mathrm{y}_{\mathrm{C}}\right)$ found earlier. Two features that are most consistent in the patterns are the trapezoidal window or tab for viewing the meniscus and the dark bank that occurs horizontally across the image previously used in the initial estimation ofy .

A binary image called the tab mask is generated from the nominal starburst pattern defining a rectangular area around the trapezoid window as seen in Figure 6. A pixel-based Boolean AND operation is performed using the tab mask and the binary pattern. In the resulting image, the trapezoid window width will have grown narrower if the hohlraum is misaligned right or left. Likewise, the trapezoid window height will be narrowed if the hohlraum is misaligned up or down. Careful measurement of the distance from the top of the trapezoid $y^{\mathrm{t}}$ to the horizontal center of the pattern yields the horizontal offset given by

$$
\Delta y=\left|y_{c}-y^{t}\right| \text {. }
$$

The distance from the center of the trapezoid $\mathrm{x}^{\mathrm{t}}$ to the vertical center of the pattern yields the vertical offset given by

$$
\Delta x=\left|\mathrm{x}_{\mathrm{c}}-\mathrm{x}^{\mathrm{t}}\right|
$$

A complete view of the various steps of the iteration loop in the alignment algorithm are depicted in the block diagram of Figure 7. 


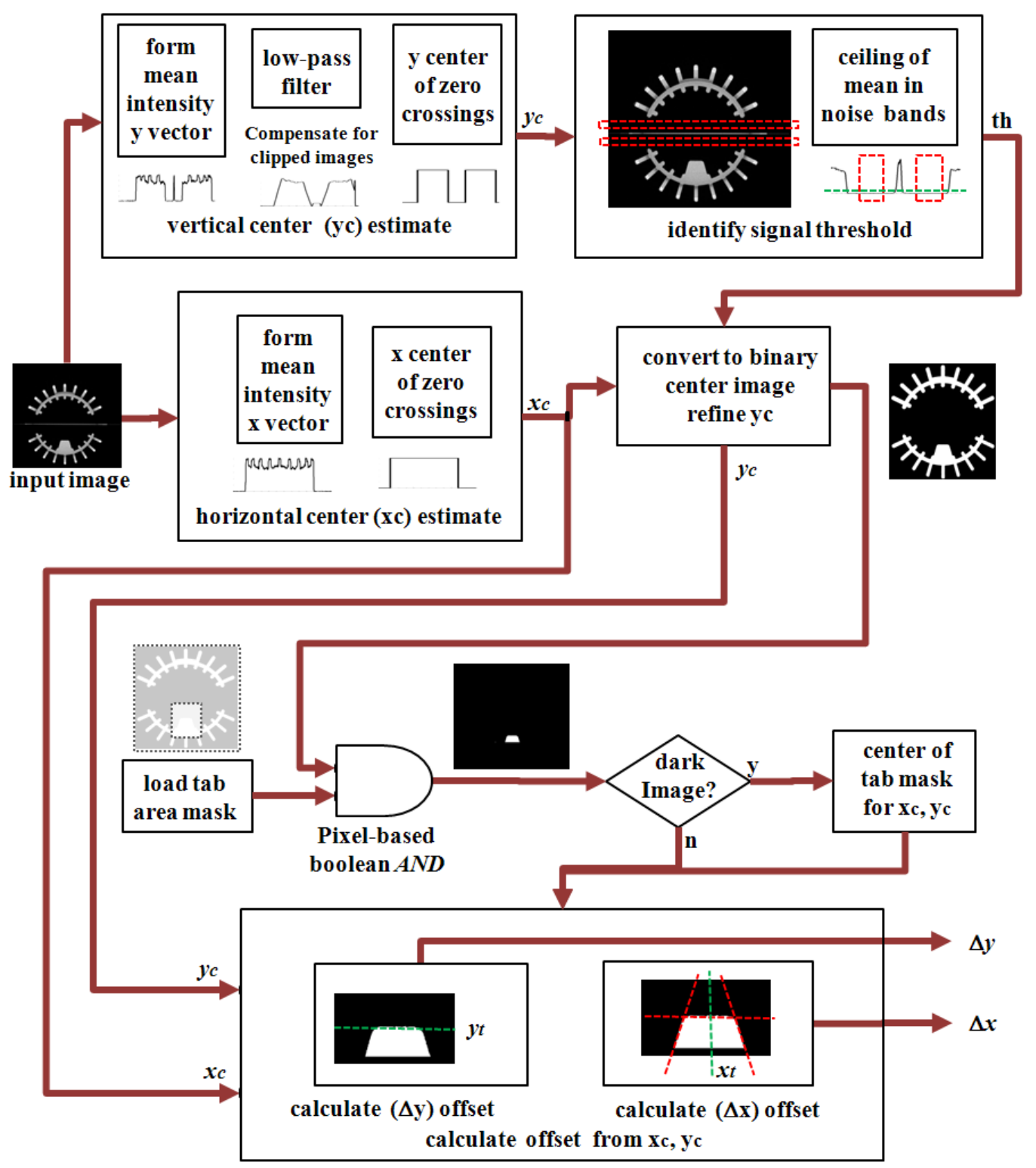

Figure 7. Block diagram of one iteration of the starburst alignment algorithm.

\subsection{Iteration required for hohlraum alignment}

The calculated offset values of $\Delta x$ and $\Delta y$ are magnitude estimates. Direction cannot be determined directly due to systematic patterns that occur twice. For example, a rear shifted starburst window can exactly match a front shifted starburst window and are indistinguishable. These effects are not separable and therefore the hohlraum is moved to four different locations denoted by $\left(\Delta x_{v} \Delta y\right)_{v}\left(-\Delta x_{v} \Delta y\right)_{v}\left(\Delta x_{v}-\Delta y\right)_{v}\left(-\Delta x_{v}-\Delta y\right)$. At each location, the binary image is calculated and integrated. The location with the largest area of integrated white pixels is used to reposition the hohlraum and the next iteration is started from the new position.

By using binary templates generated from nominal starburst patterns, we can easily generate images with desired alignment offsets by shifting one pattern with respect to a reference pattern and performing a pixel-based Boolean AND. These images can be generated to test algorithm performance. One example of this process is shown in Figure 8 where the initial offset was $\Delta x=57$ and $\Delta y=-138$ (pixels). The superposition of front to rear windows reveals only a tiny 
speck of where the $\mathrm{x}$-rays were able to penetrate. After four iterations, the final suggested move was $\Delta x=97$ and $\Delta y=28$ (pixels) and the result at the far right indicates the accurate alignment of the hohlraum.
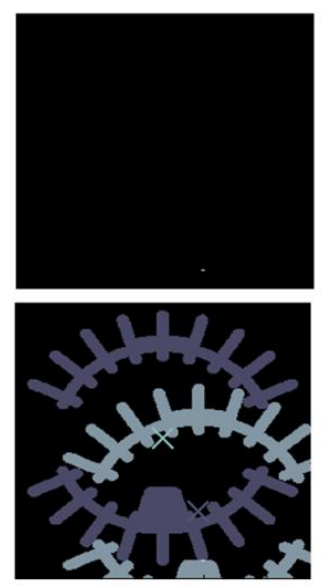

offset $\Delta x 57, \Delta y-138$

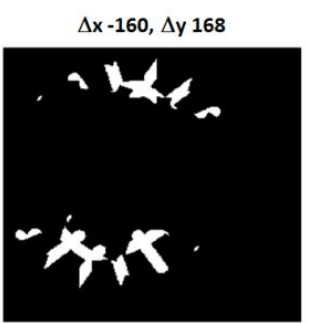

Shift left 160 pixels, up 168 pixels

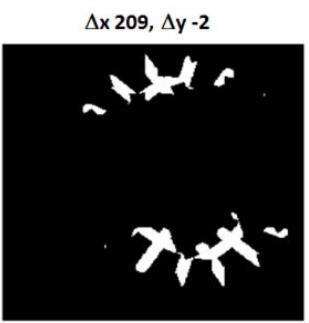

Shift right 209 pixels, down 2 pixels

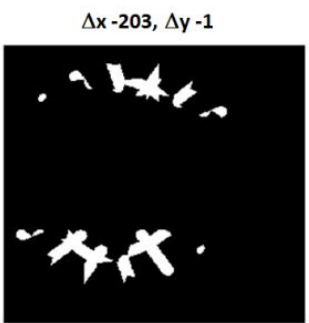

Shift left 203 pixels, down 1 pixels

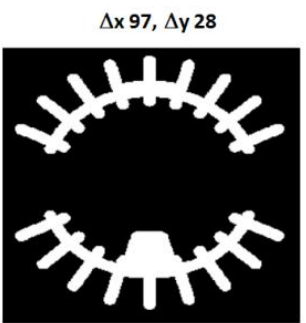

Shift right 97 pixels, Up 28 pixels

Figure 8. Original hohlraum starburst alignment position pattern is shown at left and then (left to right) after first repositioning second, third, and final position.

Using this method, we tested all shifts with $\Delta x$ varying \pm 235 pixels and $\Delta y$ varying \pm 255 pixels for a total of more than 235,000 positions. In all cases the algorithm converged. Figure 9 shows a plot of $\Delta x$ vs. $\Delta y$ vs. total iterations for all starting positions. Table 1 further illustrates that all positions achieve alignment in 15 iterations or less and nearly $98 \%$ of all starting positions achieve alignment within 5 iterations.

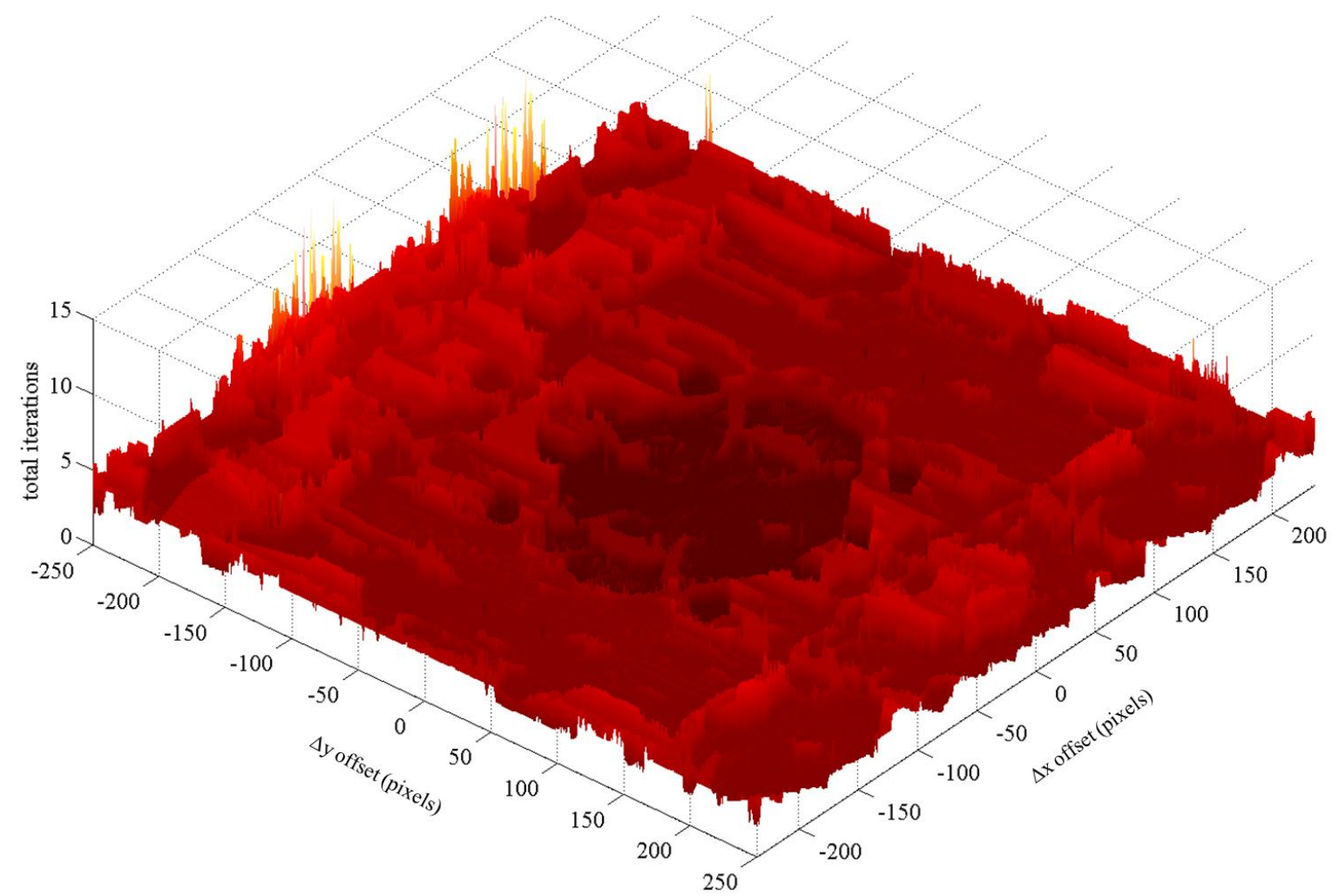

Figure 9. Plot of iterations vs. misalignment indicates that the algorithm converges for all positions. 


\begin{tabular}{|c|c|c|c|c|c|c|c|c|c|c|c|c|c|c|}
\hline 1 & 2 & 3 & 4 & 5 & 6 & 7 & 8 & 9 & 10 & 11 & 12 & 13 & 14 & 15 \\
\hline \multicolumn{15}{|c|}{ number of iterations } \\
\hline 9.219 & 35.181 & 25.625 & 20.733 & 6.892 & 1.586 & 0.464 & 0.183 & 0.067 & 0.022 & 0.012 & 0.006 & 0.003 & 0.003 & 0.005 \\
\hline \multicolumn{15}{|c|}{ fraction of total positions (\%) } \\
\hline 9.22 & 44.40 & 70.02 & 90.76 & 97.65 & 99.24 & 99.70 & 99.88 & 99.95 & 99.97 & 99.98 & 99.99 & 99.99 & 99.99 & 100 \\
\hline \multicolumn{15}{|c|}{ cumulative fraction of total positions (\%) } \\
\hline
\end{tabular}

A desired property for most iterative solutions is that if starting values are close to the solution, then the algorithm will quickly converge to the desired outcome. As seen in Figures 9 and 10 we have demonstrated that, in general, the initial position is a good indicator of performance in that the smaller starting values of $\Delta x$ and $\Delta y$, result in fewer iterations, usually less then 7, particularly within 50 pixels from hohlraum alignment. Figure 10 displays plots of each iteration number and the starting offset positions that resulted in each iteration total.
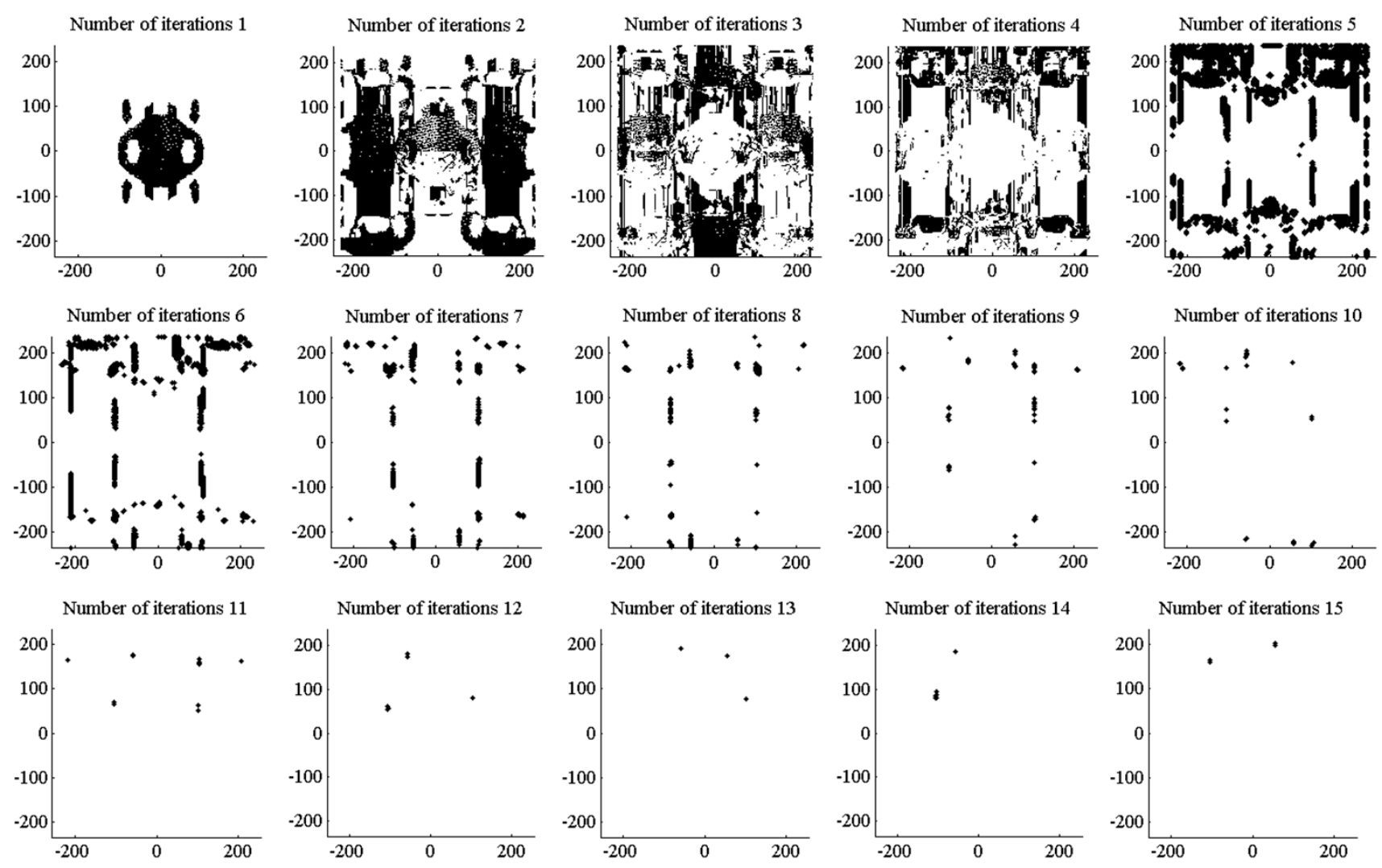

Figure 10. Hohlraum alignment completes within a few iterations with nominal starting conditions. Larger misaligned starting conditions iterate longer, but ultimately converge. 


\section{SUMMARY}

In this paper, we described the NIF target x-ray imaging system consisting of the target alignment hardware, loop control software, and underlying image processing designed to automatically align hohlraums for viewing and evaluating the DT ice layer in the target capsule. Image processing algorithms measure key features of the starburst pattern center and meniscus window location in the x-ray image to estimate a new positioning of the hohlraum. A feedback loop controls adjustment of the hardware positioning and the process is iterated until the front and back starburst windows align. The process was tested for all starting positions and found that it converged in all cases. Furthermore, when the starting position was close, as is expected in practice, less than two iterations brought the system into alignment.

\section{ACKNOWLEDGEMENT}

This work performed under the auspices of the U.S. Department of Energy by Lawrence Livermore National Laboratory under Contract DE-AC52-07NA27344. We would like to thank Steve Azevedo and Hema Chandrasekaran for their many helpful comments.

\section{REFERENCES}

1. E. I. Moses, The National Ignition Facility and the National Ignition Campaign", Vol. 38 No. 4, IEEE Transactions on Plasma Science, Apr, 2010

2. E. I Moses, "Ignition on the National Ignition Facility: a path towards internal fusion energy", 49, Nuclear Fusion, IOP Publishing and International Atomic Energy Agency, Sep, 2009

3. C. A. Haynam, P. J. Wegner, G. M. Heestand, E. Moses, R. A. Sacks, M. W. Bowers, S. N. Dixit, G. V. Erbert, M. A. Henesian, M. R. Hermann, K. S. Jancaitis, K. Knittel, T. Kohut, K. R. Manes, C. D. Marshall, N. C. Mehta, J. Menapace, J. R. Murray, M. C. Nostrand, C. D. Orth, R. Patterson, R. Saunders, M. J. Shaw, M. Spaeth, and S. B. Sutton, "The National Ignition Facility: Status and Performance of the World's Largest Laser System for the High Energy Density and Inertial Confinement Fusion," in Conference on Lasers and Electro-Optics/Quantum Electronics and Laser Science Conference and Photonic Applications Systems Technologies, OSA Technical Digest (CD) (Optical Society of America, 2008), paper CFQ1.

4. S. H. Glenzer', B. J. MacGowan,, P. Michel, N. B. Meezan, L. J. Suter, S. N. Dixit, J. L. Kline, G. A. Kyrala, D. K. Bradley, D. A. Callahan, E. L. Dewald, L. Divol ${ }^{1}$, E. Dzenitis, M. J. Edwards, A. V. Hamza, C. A. Haynam, D. E. Hinkel, D. H. Kalantar, J. D. Kilkenny, O. L. Landen, J. D. Lindl, S. LePape, J. D. Moody, A. Nikroo, T. Parham, M. B. Schneider, R. P. J. Town, P. Wegner, K. Widmann, P. Whitman, B. K. F. Young, B. Van Wonterghem, L. J. Atherton and E. I. Moses, "Symmetric Inertial Confinement Fusion Implosions at Ultra-High Laser Energies", Vol. 327 no. 5970 pp. 1228-1231, Science, January, 2010

5. Edward I. Moses, "Advances in inertial confinement fusion at the National Ignition Facility", 85, p983-986, Fusion Engineering and Design, Jan, 2010

6. B. J. Kozioziemski, S. O. Kucheyev, J. B. Lugten, J. A. Koch, J. D. Moody, A. A. Chernov, E. A. Mapoles, A. V. Hamza, and L. J. Atherton, "Plastic deformation of solid hydrogen in fusion targets", AIP Journal of Applied Physics, 105, (2009)

7. C. Gibson, J. Baltz, T. Malsbury, D. Atkinson, V. Brugmann, F. Coffield, O. Edwards, B. Haid, S. Locke, S.shiromizu, K. Skulina, "Design of the NIF Cryogenic Target System", LLNL, Fusion Science and Technology, LLNL-JRNL-404974, June, 2008

8. D. Marr. Vision. Freeman, 1982.

9. D. Marr and E. C. Hildreth. Theory of edge detection. Proceedings of the Royal Society, London B, 207:187-217, 1980

10. J. F. Canny. Finding edges and lines in images. Master's thesis, MIT. AI Lab. TR-720, 1983.

11. J. F. Canny. A computational approach to edge detection. IEEE Trans. Pattern Analysis and Machine Intelligence, 8:679-698, 1986.

12. Rachid Deriche. Using Canny's criteria to derive an optimal edge detector recursively implemented. The International Journal of Computer Vision, 1:167-187, April 1987.

13. Margaret M. Fleck. Multiple widths yield reliable finite differences. IEEE Transactions PAMI, 14(4): 412-429, April 1992.

14. Bidut Baran Chaudhuri and Bhabatosh Chanda, "The equivalence of best plane fit gradient with Robert's Prewitt's and Sobel's gradient for edge detection and a 4-neighbor gradient with useful properties", Signal Processing 6 , p143-151 1984 